I $\propto$ RADIATION AND CaII IONIZATION IN THE TYPE II SUPERNOVAE AT LATE TIMES

\title{
Nikolai N.Chugai
}

Astronomical Council of the Academy of Sciences of the USSR Pjatnitskaja 48, Moscow 109071, USSR

Abstract: The ionization of CaII by the $I \alpha$ quanta in the envelope of the type II supernova $1970 \mathrm{~g}$ on 270 th day is considered. The ratio CaII/CaIII is found to be very low $(<0.1)$. This results in the low theoretical intengities of the CaII emission lines; they are at least an order of magnitude weaker than the observed ones. The implications are discussed.

An interpretation of the strong[CEII] $\lambda 7300$ and $\lambda 8600$ emissions in the late time spectra of SNII, e.g. SN1970g on 270 th day after the explosion (see: Kirshner et al., 1973; Kirshner and Kwan, 1975) is related closely to the value of CaII fractional ionization. Yet the situation with the ratio CaII/Ca is indefinite because the contribution of I $\propto$ quanta into the ionization of CaII has been ignored so far. This process is studied here for the case of SN1970g on 270 th day.

A following simple model for the envelope is adopted (see: Chugai, 1987): a homogeneous sphere with the expansion law $v=r / t$, boundary velocity $\mathrm{v}_{0}=4000 \mathrm{~km} \mathrm{~s}{ }^{-1}$, hydrogen mass $\mathrm{M}_{\mathrm{H}_{7}}=2.5 \mathrm{M}_{0}$ (total mass is $\approx 4 M_{\odot}$ ), electron concentration $\mathrm{n}_{e}=3.5 \cdot 10^{7} \mathrm{~cm}^{-3}$, electron temperature $\mathrm{T}_{\mathbf{e}}=7000 \mathrm{~K}$. The hydrogen population on the second level is $\mathrm{n}_{2}>7 \mathrm{~cm}^{-3}$ (Kirshner and Kwan, 1975; Chugai, 1987).

I $\propto$ quanta ionize CaII from $3 d$ level $\left(\lambda_{3 \mathrm{~d}}=1218 \AA\right)$. The density of $L \propto$ quanta with $\lambda<\lambda_{3 d}$ at some point inside the envelope is determined by their creation and scattering in the local region of $a$ "sound" radius $v_{t h} t \ll v_{0} t$. The $L \propto$ spectrum is specified by: the local optical depth $\tau=\mathrm{k} \lambda t=2.5 \cdot 10^{9}$, Voight parameter $\mathrm{a}=5.6 \cdot 10^{-4}$, collisional destruction probability $\varepsilon=C(2 \mathrm{p}, 2 \mathrm{~s}) / \mathrm{A}(2 \mathrm{p}, 1 \mathrm{~s})=10^{-5}$, continuous absorption parameter $\omega_{c}=k_{c} \Delta \nu_{D} / k \leqslant 10^{-12}$. In the selective absorption of $L \propto$ the most pronounced effect is produced by FeII $\lambda 1217.85$ line with the absorption parameter $\omega_{\mathbf{s}}=\mathrm{k}_{\mathbf{s}} / \mathrm{k} \approx 2 \cdot 10^{-9}$. For the parameters indicated above the rate of CaII photoionization with the $L \propto$ quanta is found to be $P_{3 d}>0.020\left(M_{H} / 2.5 M_{\odot}\right) s^{-1}$. 
The photoionization balance of CaII in the SN1970g on 270 th day is calculated in the "two level (4s and $3 d$ ) plus continuum" approximation. A lower limit for $\mathrm{P}_{3 \mathrm{~d}}$ and the solar ratio for $\mathrm{Ca} / \mathrm{H}$ are adopted. We found that: (1) the ionization of CaII with $L \alpha$ radiation in $S N$ $1970 \mathrm{~g}$ on 270 th day is the very effective process which results in CaII/CaIII < 0.1 ; (2) the theoretical intensity of [CaII] $\lambda 7300$ emission is at least an order of magnitude lower than the observed one. The latter refers to CaII $\lambda 8600$ as well. Both results are stable against variation of the hydrogen mass in the range $1.5+3.5 \mathrm{M}$.

The discrepancy between predicted and observed intensities of the CaII emissions in the case of $5 N 1970 \mathrm{~g}$ on 270 th day requires some modification of the model applied above. Three possibilities are conceivable.

1) $\mathrm{Ca}$ is overabundant, i.e. $z_{S N}(\mathrm{Ca}) \approx 10 \mathrm{z}_{\mathrm{o}}(\mathrm{Ca})$.

2) The CaII fractional ionization is controlled with the charge exchange $\mathrm{Ca}^{++}+\mathrm{H}+1.7 \mathrm{eV} \rightleftharpoons \mathrm{Ca}^{+}+\mathrm{H}^{+}$which results in CaII/CaIII $\approx$ 1.5 provided the cross-section for the reaction from the right to the left $\sigma \geqslant 2 \cdot 10^{-17} \mathrm{~cm}^{2}$. Unfortunately the value of $\sigma$ is not known.

3) The SN1970g envelope is chemically inhomogeneous and consist of the two main components: (a) H-rich gas and (b) He-rich filaments which are inserted into the H-rich background. GaII emission lines presumably originate from He-rich component where $\mathrm{Ca}$ is ionized singly. To account for the observed CaII emission an order of $1 \mathrm{M}_{\circ}$ in the form of He-rich filaments is required. Such inhomogeneous structure might be produced by the Rayleigh-Taylor instability during the supernova explosion when the H-rich red supergiant envelope is shocked with the fast expanding He-rich matter.

The third possibility is favoured by the absence of the strong absorption component of $H \propto$ in the spectra of SN1970g at earlier phase which is consistent with the idea of the inhomogeneous distribution of the hydrogen in the supernova envelope (see: Chugai, 1982).

\section{References}

Chugai N.N. 1982, Astron. Zh., 29, 1134.

Chugai N.N. 1987, Pis'ma Astron. Zh., 13, 671.

Kirshner R.P., Oke J.B., Penston M.V., Searle I. 1973. Ap. J., $185,303$.

Kirshner R.P. and Kwan J. 1975, Ap. J., 197, 415. 